\title{
Referate und Rezensionen
}

\section{Referate}

\section{Die neuen amerikanischen Leitlinien zur Ernährung von kritisch kranken Patienten}

Taylor B. E. et al. Guidelines for the Provision and Assessment of Nutrition Support Therapy in the Adult Critically III Patient: Society of Critical Care Medicine (SCCM) and American Society for Parenteral and Enteral Nutrition (A.S.P.E.N.). Journal of Parenteral and Enteral Nutrition 2016: 40: 159-211

Die amerikanische Society of Critical Care Medicine (SCCM) und die American Society for Parenteral and Enteral Nutrition (A.S.P.E.N.) hatten sich zum Ziel gesetzt, ca. alle 5 Jahre gemeinsame Leitlinien zur Ernährung von kritisch kranken Patienten (i.d.R. Intensivpatienten) zu publizieren. Die letzte dieser Leitlinien war von 2009, sodass nun die Zeit für eine Aktualisierung gekommen ist. In den erst in diesem Jahr erschienenen Guidelines wurde nur Literatur berücksichtigt, die bis zum 31.12.2013 publiziert wurde. Die Studienzusammenstellung und die Aussagen der Leitlinie zielten auf Patientengruppen über 18 Jahren und mit einem Intensivaufenthalt länger als 2-3 Tagen ab.

Die Empfehlungen lassen sich in 6 Hauptempfehlungen zusammenfassen:

1. Der Ernährungsstatus von Patienten sollte unmittelbar nach Aufnahme auf die Intensivstation erfasst werden, möglichst mit Scores, die speziell für diese intensivmedizinische Ernährungserfassung von kritisch kranken Patienten erstellt wurden.

2. Eine enterale Ernährung sollte während der ersten 24-48 Stunden nach Aufnahme auf die Intensivstation gestartet und dann schrittweise innerhalb der ersten Woche gesteigert werden.

3. Es sollten Maßnahmen durchgeführt werden, die das Risiko von Aspiration bzw. Malresorption verringern.

4. Es sollten speziell auf die jeweilige Intensivstation angepasste Protokolle zur Verbesserung der enteralen Ernährung erstellt werden.

5. Das Residualvolumen der enteralen Ernährungslösung im Magen sollte nicht zur Steuerung der enteralen Ernährung herangezogen werden.

6. Parenterale Ernährung sollte früh gestartet werden, wenn bei unterernährten Patienten eine ausreichende enterale Ernährung nicht möglich ist.

Neben diesen 6 Hauptempfehlungen, die zu einem Handlungsbündel zusammengefasst wurden, finden sich eine Reihe von weiteren wichtigen Empfehlungen, u.a. die Empfehlung, die erforderliche Ernährungsdosis mithilfe der indirekten Kalorimetrie zu bestimmen oder, wenn das nicht möglich ist, ein Ernährungsziel von 25-30 kCal/kgKG/Tag zu erreichen. Eine enterale Ernährung über eine Magensonde ist bei den meisten Patienten ausreichend. Nur bei Patienten mit hohem Aspirationsrisiko sollte eine möglichst distale enterale Ernährung versucht werden. Bei hämodynamischer Instabilität sollte keine enterale Ernährung durchgeführt werden. Es sollte eine HochdosisProteingabe mit 1,2-2,0 g/kgKG/Tag erfolgen, primär Standardernährungslösungen benutzt werden und keine Krankheitsbild-spezifischen Lösungen. Glutamin sollte weder in enteralen noch in parenteralen Lösungen zusätzlich zugeführt werden.

Auch wenn die Ergebnisse großer multizentrischer Studien der letzten 2 Jahre einen Teil der Empfehlungen dieser Leitlinie in Frage stellen, so ist doch das Lesen dieser Empfehlungen sinnvoll, um das
Handeln auf der eigenen Intensivstation noch einmal zu überdenken.

Fazit Neue Leitlinien zur enteralen und parenteralen Ernährung bei Intensivpatienten sind erschienen.

\section{Ist die „vermeintlich“ physio- logische Kochsalzlösung gefährlich bei „quasi gesunden“ Intensivpatienten? \\ Young P. et al. Effect of a Buffered Crystal- loid Solution vs Saline on Acute Kidney Injury Among Patients in the Intensive Care Unit. The SPLIT Randomized Clinical Trial. JAMA 2015; 314: 1701-1710}

Die Diskussion über die Gefahren bzw. den Nutzen von HAES-Lösungen bei Intensivpatienten wurde in den letzten 10 Jahren mit großer Intensität geführt. Etwas aus den Augen verloren wurde hierbei die schon wesentlich ältere Diskussion über Gefahren einer „physiologischen“ Kochsalzlösung zur Volumentherapie bei kritisch kranken Patienten. Neben dem Entstehen einer hyperchlorämen Azidose durch die unphysiologisch hohen Chloridkonzentrationen in den $\mathrm{NaCl}$-Lösungen wird auch seit längerer Zeit das Risiko eines akuten Nierenversagens durch die „physiologischen“ Kochsalzlösungen diskutiert. Neben den NaCl-Lösungen stehen als kristalloide Lösungen auch gepufferte Elektrolytlösungen mit einer Elektrolytzusammensetzung zur Verfügung, die der Zusammensetzung im menschlichen Blut näher kommt. Zu diesen Lösungen gehört u.a. Ringer-Laktat-Lösung. In einer großen neuseeländischen Multicenterstudie wurden aktuell die Effekte von NaCl-0,9\%Lösungen mit gepufferten Elektrolytlösungen verglichen. Hierbei war der primäre Outcomeparameter das Auftreten eines akuten Nierenversagens. Insgesamt wurden 2278 Patienten in die Studie ein- 
geschlossen und in die beiden Gruppen randomisiert. Die meisten der eingeschlossenen Patienten wurden nach elektiver Operation auf die Intensivstation verlegt. Fast 90\% der eingeschlossenen Patienten hatten vor Aufnahme auf die Intensivstation schon gepufferte Elektrolytlösungen erhalten. Der mittlere APACHE II-Score der Patienten war mit 14 eher niedrig. Die mittlere Aufenthaltsdauer auf der Intensivstation betrug nur 1,5 Tage. Während dieses kurzen Intensivaufenthaltes erhielten die Patienten in beiden Behandlungsgruppen ca. $2000 \mathrm{ml}$ Kristalloid-Lösung. Zwischen der Gruppe mit NaCl-Lösung und der Gruppe mit balancierter Elektrolytlösung fanden sich in keinem der untersuchten Parameter signifikante Unterschiede, u.a. fanden sich keine Unterschiede in

- der Häufigkeit von akutem Nierenversagen,

- den Kreatininwerten,

- der Häufigkeit einer metabolischen Azidose und

- der Mortalität.

Zusammenfassend hat die Studie von Young et al. gezeigt, dass, wenn man geringe Mengen an unterschiedlichen Elektrolytlösungen an Intensivpatienten gibt, die eine geringe Krankheitsschwere aufweisen und sich auch nur sehr kurz auf der Intensivstation aufhalten, sich keine signifikanten Unterschiede im Outcome finden. Erneut fragt man sich, wie solche klinisch wenig relevanten intensivmedizinischen Studien ihren Weg in ein hochrangiges Journal wie das JAMA finden.

Fazit Geringe Mengen an „physiologischer" Kochsalzlösung scheinen während eines kurzen Intensivaufenthaltes von relativ gesunden Patienten nicht schädlich zu sein.

\section{Die Ultraschall-gesteuerte Dilatationstracheotomie ist im Kommen!}

\section{Gobatto A. L. N. et al. Ultrasound-guided percutaneous dilational tracheostomy versus bronchoscopy-guided percutaneous dilational tracheostomy in critically ill patients, (TRACHUS): a randomized non- inferiority controlled trial. Intensive Care Med 2016; 42: 342-351}

Die perkutane Dilatationstracheotomie hat sich innerhalb der letzten 2 Jahrzehnte zu einem Standardverfahren auf der Intensivstation entwickelt. Sie ist im Vergleich zur chirurgischen Tracheotomie mit einem geringeren logistischen Aufwand und mit einem besseren Sicherheitsprofil durchzuführen. Bislang wird auf den meisten Intensivstationen die Dilatationstracheotomie unter bronchoskopischer Kontrolle durchgeführt. Mithilfe der Bronchoskopie kann die korrekte Punktionsstelle verifiziert, eine Tracheahinterwandläsion vermieden und das korrekte Vorschieben des Führungsdrahtes und später die korrekte Tubuslage beurteilt werden. Da durch eine Bronchoskopie weder prätracheale größere Gefäße oder Schilddrüsenstrukturen festgestellt werden können, kombinieren viele Kliniken mittlerweile die Bronchoskopie mit einer Ultraschalluntersuchung vor Beginn der Tracheotomie. In einzelnen aktuellen kleineren Studien wurde nun die ausschließlich Ultraschall-gesteuerte Dilatationstracheotomie mit einer ausschließlich bronchoskopisch gesteuerten Dilatationstracheotomie verglichen. Die Aussagekraft dieser Studien war jedoch wegen des Studiendesigns und der geringen Fallzahl nur sehr eingeschränkt. In einer singlezentrischen, randomisierten Studie, die aber auf 8 verschiedenen Intensivstationen des Klinikums in Sao Paulo durchgeführt wurde, haben die Autoren nun die ausschließlich ultraschallgesteuerte Tracheotomie mit der ausschließlich bronchoskopisch geführten Tracheotomie verglichen. (Methoden im Online-Supplement J Crit Care 30:220.e213-220.e227). Insgesamt wurden 118 Patienten randomisiert für diese 2 unterschiedlichen Tracheotomieverfahren. Die mittlere Dauer der Tracheotomie unterschied sich nicht signifikant zwischen beiden Verfahren (11 Minuten vs. 13 Minuten). Bei einem Patienten in der Ultraschallgruppe und bei einem Patienten in der Bronchoskopiegruppe war technisch die Dilatationstracheotomie nicht durchführbar. In beiden Gruppen trat jeweils einmal eine schwere Komplikation auf: Bei einem Patienten in der Ultraschallgruppe wurde zu tief punktiert mit nachfolgender Trachealläsion und Mediastinitis. In der Bronchoskopiegruppe wurde bei einem Patienten mit der Dilatationsklemme die Tracheavorderwand beschädigt mit nachfolgendem Pneumomediastinum. Auch bei der Häufigkeit kleinerer Komplikationen und bei allen untersuchten Outcomeparametern fand sich kein signifikanter Unterschied zwischen den beiden Gruppen.

Fazit Eine ausschließlich durch Ultraschall gesteuerte Dilatationstracheotomie ist nicht mit mehr Komplikationen oder einem schlechteren Outcome verbunden als eine rein bronchoskopisch kontrollierte Dilatationstracheotomie.

\section{Expertenmeinungen zum akuten Herzversagen und zum kardiogenen Schock}

\section{Mebazaa A et al. Acute heart failure and cardiogenic shock: a multidisciplinary practical guidance. Intensive Care Med 2016; 42: 147-163}

Das GREAT-Network ist ein lockeres internationales Netzwerk zur Förderung der präklinischen und innerklinischen Versorgung von Notfallpatienten. Eine Expertengruppe aus diesem Netzwerk hat nun Empfehlungen (Guidance) zur Diagnose und zur Therapie von akutem Herzversagen und kardiogenem Schock publiziert. Diese 22 Experten stammten aus unterschiedlichen Ländern von allen 5 Kontinenten und aus unterschiedlichen medizinischen Fachrichtungen wie z.B. Kardiologie, Intensivmedizin, Notfall- 
medizin, Anästhesie oder Allgemeine Innere Medizin. Da aktuelle internationale Leitlinien zur Behandlung des akuten Herzversagens und des kardiogenen Schocks fehlten, haben die Autoren ihre Empfehlungen publiziert. Diese Empfehlungen sind besonders für die Therapie auf der Intensivstation vorgesehen. Verwunderlicher Weise fehlt im Intensiv-CareArtikel mit diesen Empfehlungen jeglicher Methodenteil, sodass es nicht möglich ist nachzuvollziehen, durch welches Verfahren die Autoren auf ihre Empfehlungen gekommen sind. Insgesamt enthalten die Empfehlungen der Autoren nichts wesentlich Neues - das Betonen der Bedeutung des pulmonalen Ultraschalls zur Diagnose eines Lungenödems mag vielleicht noch den einen oder anderen überraschen. Aufgrund der sehr praxisnahen Empfehlungen und der hilfreichen grafisch gut dargestellten Therapiealgorithmen ist der Artikel aber durchaus lesenswert.

\section{Fazit Ein guter Reviewartikel zur} Behandlung von akutem Herzversagen und kardiogenem Schock.

\section{Die neuen Sepsis-Definitionen: komplizierter, Outcome-relevanter?}

\section{Singer M. et al. The Third International} Consensus Definitions for Sepsis and Septic Shock (Sepsis-3). JAMA 2016; 315: 801-810

Sepsis ist eine der wichtigsten Ursachen für das Versterben von Patienten im Krankenhaus. Die Häufigkeit und die Sterblichkeit der Sepsis sind höher als z. B. bei akuten kardialen Problemen, Schlaganfall oder Traumen. Zusätzlich ist auch eine Mortalitätsreduktion durch eine frühe Behandlung der Sepsis belegt. Dennoch wird einer notfallmäßigen Behandlung der Sepsis weniger Aufmerksamkeit gewidmet als der notfallmäßigen Behandlung der oben genannten Krankheitsbilder Schlaganfall, Trauma oder kardialer Dekompensation. Bislang gab es 1991 und 2001 von verschiedenen Fachgesellschaf- ten konsentierte Sepsis-Definitionen, die jeweils dem aktuellen Stand der wissenschaftlichen Forschung angepasst waren. Aktuell wurden nun zum 3. Mal neue Sepsis-Definitionen publiziert, die dann auch konsequenterweise als Sepsis-3 benannt wurden. Für den Entwicklungsprozess dieser neuen Definitionen wurden von der Society of Critical Care Medicine und der European Society of Intensive Care Medicine 19 Experten benannt, die gemeinsam die Definitionen entwickelten. Anschließend wurden die Definitionen von über 30 internationalen Fachgesellschaften aus unterschiedlichen Bereichen der Medizin konsentiert. Ziel der neuen Sepsisdefinitionen war es, eine Sepsis von einer unkomplizierten Infektion zu differenzieren und gleichzeitig die Definitionen von Sepsis und septischem Schock den aktuelleren wissenschaftlichen Erkenntnissen anzupassen. Die neuen Definitionen sollten es niedergelassenen Ärzten, Ärzten in den Notaufnahmen, aber auch Ärzten auf Normal- und Intensivstationen ermöglichen, eine Sepsis schneller zu diagnostizieren und somit schneller eine adäquate Therapie einleiten zu können. Die Experten verwarfen im Entwicklungsprozess schnell die ehemaligen SIRSKriterien der 1991-Definitionen, da die SIRS-Kriterien bei vielen Patienten ohne Infektionen und ohne nachfolgende Komplikationen erfüllt werden und hingegen viele Intensivpatienten mit Infektionen und Organversagen die SIRS-Kriterien nicht erfüllen. Die generelle neue Definition der Sepsis lautet nun: Sepsis ist ein lebensbedrohliches Organversagen aufgrund einer inadäquaten Reaktion des Körpers auf eine Infektion (,life-threatening organ dysfunction caused by a dysregulated host response to infection“). Mit dieser Definition wurde der zuvor bestehende Begriff „schwere Sepsis“ überflüssig und ist auch nicht mehr vorgesehen. Zur Bestimmung des Organversagens nutzen die Autoren den SOFA-Score (Sequential [Sepsis-Related] Organ Failure Assessment Score). Bei einem Anstieg dieses Scores um 2 oder mehr Punkte im Verlauf einer nachgewiesenen oder vermuteten Infektion liegt dann eine Sepsis vor. Bei
Patienten ohne zuvor bestehendes Organversagen kann der SOFA-Ausgangsscore mit 0 angesetzt werden. Da außerhalb der Intensivstationen die Erhebung eines SOFA-Scores aufwendig ist und Laboruntersuchungen erfordert, wurde ein sogenannter Quick-SOFA (qSOFA) in die Definition eingefügt. In diesen qSOFA gehen nur die folgenden Parameter ein:

- veränderter Bewusstseinsstatus

- systolischer Blutdruck $\leq 100 \mathrm{mmHg}$

- Atemfrequenz $\geq 22 / \mathrm{min}$.

Außerhalb der Intensivstation soll dieser qSOFA genutzt werden und bei 2 oder mehr erfüllten Parametern ein klassischer SOFA-Score erhoben werden. Ist dieser dann $\geq 2$ Punkte höher als der Ausgangswert und liegt zusätzlich eine vermutete oder nachgewiesene Infektion vor, kann wie oben schon erwähnt, von einer Sepsis ausgegangen werden. Auch der septische Schock wurde neu definiert als:

1. Sepsis,

2. Vasopressorpflicht, um bei Normovolämie den mittleren arteriellen Blutdruck über $65 \mathrm{mmHg}$ zu halten,

3. Laktatwerte $>2 \mathrm{mmol} / \mathrm{l}$.

Am schönsten und einfachsten kann die neue Sepsisdefinition anhand des Algorithmus, der in einer Abbildung im frei verfügbaren Artikel dargestellt ist, nachvollzogen werden. Die neuen Sepsis-Definitionen sind anfänglich sicherlich noch ungewohnt, weil sie u.a. auf der Intensivstation die Erfassung des SOFA-Scores voraussetzen, außerhalb der Intensivstationen kann aber der qSOFA zu einer schnelleren Sepsis-Diagnose und nachfolgend auch zu einer schnelleren Therapie führen. Sind diese neuen Definitionen komplizierter? Am Anfang sicherlich, aber sicherlich auch besser für ein schnelleres Agieren und damit auch ein besseres Outcome.

Fazit Die neuen internationalen Definitionen für den Begriff Sepsis und septischer Schock sind da und sollten auch benutzt werden. 